\title{
STUDY ON THE CHARACTERISTICS OF BEIJING SUBSIDENCE BASED ON PS- INSAR/LEVELING AND PRIMARY INVESTIGATION OF THE RELATIONSHIP WITH FAULT ZONE
}

\author{
WANG Xiaoqing, ZHANG Peng, WANG Yongshang, SUN Zhanyi \\ Dept of Geodesy, National Geomatics Center of China, China-(xqwang, zhangpeng, szy, wys)@ngcc.cn
}

KEY WORDS: Beijing land subsidence, PS-InSAR, levelling, features study, fault zone.

\begin{abstract}
:
The severe land subsidence could lead to ground collapse, building damage and a series of disasters. Up to now, the land subsidence has occurred in more than 50 cities in China, which seriously affects the life and production safety of local people and restricts the development of cities. While, Beijing is one of the most serious cities. This paper takes the urban area of Beijing as an example. PSInSAR technology is used to process 40 scenes of Terra SAR images from 2010 to 2015, and the high-coherence points are selected by fusing the two algorithms of coherence coefficient and amplitude deviation. In order to verify the reliability of the results, the second-level measurement results are compared with the PS-InSAR deformation results, and five leveling points are used to evaluate the accuracy. The results show that: the maximum absolute error between the Leveling results and the InSAR measurement result is $8.87 \mathrm{~mm}$, and the standard error is $3.22 \mathrm{~mm}$, which meets the accuracy requirements. And areas with serious subsidence occur in Changping District, Haidian District, Daxing District, and Chaoyang District; there is no obvious subsidence trend in the central and eastern parts of Dongcheng, Xicheng and Fengtai District, and the surface is relatively stable. The subsidence in Tongzhou and Shunyi District is serious relatively, the subsidence in these two areas is $-6 \mathrm{~mm} / \mathrm{a} \sim-67 \mathrm{~mm} / \mathrm{a}$ and $-11 \mathrm{~mm} / \mathrm{a} \sim-22 \mathrm{~mm} / \mathrm{a}$ respectively. Finally, the spatial relationship between fault zones and land subsidence was preliminarily discussed. The results show that, the subsidence of the south of Changping was so serious than others while the Nankou-Sunhe fault zone crossed with Babaoshan and HuangzhuangGaoliying fault zone through this area respectively.
\end{abstract}

\section{INTRODUCTION}

Land subsidence refers to a falling movement that local strata surface elevation drops due to compression of crusted soil under the impact of human activities and natural factors, which is characterized by imperceptibility, wide impact scope, and irreversibility[1][2][3]. Severe land subsidence will cause land collapse, building damage and a series of disasters. As of 2011, land subsidence has occurred in more than 50 cities of China, which greatly affected local people's lives and production safety and restricted cities' development[4][5].

Conventional land subsidence monitoring methods include precise leveling and GPS measuring, which cannot satisfy the need of wide-range land subsidence monitoring of high spatiotemporal resolution for the disadvantages of high cost and longtime-interval of measurement[6].

Differential interferometric synthetic aperture radar (D-InSAR) can be applied for wide-range all-weather terrain monitoring of high resolution, having become the most effective approach of urban land subsidence monitoring[7]. Solari, by combining geological and geotechnical data with PS-InSAR data, depicted the characteristics of temporal and spatial variation of land subsidence of Pizza Plains in Italy, and concluded that the main causes of land subsidence in this area were high compressibility of clay stratum and the nearest urbanization to the east of the city [8]. Zhu et al. monitored the northern part of Beijing for subsidence by virtue of ASAR data in combination of StaMPS technology, and concluded that no significant correlation existed between urban development and subsidence rate[9]. Yang et al. employed PS-InSAR technology to investigate the correlation between land subsidence and buildings in the urban area of the eastern part of Beijing, and found that dense building constructions in city might cause massive differential settlement, and settlement of individual structures might be impacted by architectural volume, basic structure and hydrogeological conditions[10].

For the case study of the urban area of Beijing, we gathered TerraSAR data of 40 scenes in this area. The data is of high spatio-temporal resolution, and sufficient PS points can be obtained based on it to reduce distanglement error. Besides, the wave length of $\mathrm{X}$ waveband is short, and more sensitive to deformation. The PS-InSAR technology was adopted to conduct temporal subsidence monitoring over the urban area of Beijing to gather subsidence information, and focus was placed on analysis of subsidence variation trend of the urban area of Beijing and the distribution characteristics and of the spatial relationship between land subsidence and fault zones.

\section{METHODOLOGY}

Over the past decades, time-series InSAR processing algorithms have been developed step by step. In 2000, Persistent Scatterers InSAR (PSInSAR) was proposed, which had been proven as an effective tool for deformation multi-temporal analysis in urban areas. Considering the normal distribution of the PSs' amplitude, the amplitude dispersion index was commonly used as the criterion for PSs selection. Based on these discrete pixels, spatial-temporal filter was employed to separate deformation phase from other phase components. However, it will neglect some points in vegetation which are coherent temporarily. PS InSAR is a kind of radar Differential Interferometry Technology Based on single main image, which establishes a 
function model for the points with high coherence in time series SAR image to obtain the surface deformation value. Firstly, a main image is determined according to the correlation criteria. After registration and resampling, the candidate points of permanent scatterers are selected according to the amplitude deviation threshold method. The Permanent Scatterers on the main image are interfered with the corresponding points on other images to obtain the interference phase of each permanent scatterer. Then, the differential interference phase is obtained after removing the flat effect and surface elevation phase, The unwrapped phase of the first interferogram is expressed by the following formula:

$$
\Phi^{k}=\phi_{\text {topo }}^{k}+\phi_{\text {defo }}^{k}+\phi_{\text {atmo }}^{k}+\phi_{\text {noise }}^{k}
$$

Where, $\phi_{\text {topo }}^{k}$ is the phase value caused by reference phase error, $\phi_{d e f o}^{k}$ is the phase value caused by ground object moving in the radar line of sight direction, $\phi_{\text {atmo }}^{k}$ is the phase caused by atmospheric delay, and $\phi_{\text {noise }}^{k}$ is the phase of decorrelation noise. In fact, the terrain phase is a linear function of the vertical baseline, It can be expressed as:

$$
\phi_{x, \text { topo }}^{k}=\beta_{x}^{k} \cdot \Delta h_{x}
$$

$\beta_{x}^{k}$ is the conversion factor of elevation conversion to phase, and $\Delta h_{x}$ is elevation error. Assuming that deformation is linear deformation, it is also possible to describe deformation phase in linear relation with time, as follows:

$$
\phi_{x, \text { defo }}^{k}=-\frac{4 \pi}{\lambda} T^{k} \cdot \alpha(x)
$$

Where, ${ }^{\lambda}$ is the wavelength of the radar signal, ${ }^{T^{k}}$ is the time interval relative to the main image, and ${ }^{\alpha(x)}$ is the average deformation velocity of the point.

Except for the interference phase between the ground level phase and terrain elevation, there is no unwrapping, and the phase value is between, which is a nonlinear problem and cannot be solved directly. The solution space search method is needed to solve the problem. In the process of solving the problem, an important parameter, the integral phase coherence, is used, which is expressed as follows:

$$
\gamma_{x . y}=\frac{1}{N-1} \sum_{k=1}^{N-1} \exp \left(j e_{x, y}^{k}\right)
$$

Where, $j$ is the imaginary unit, and the difference between the model phase and the real phase expressed as follows:

$$
e_{x, y}^{k}=\phi_{x, y}^{k}-\left(\beta_{x}^{k} \cdot \Delta h_{x, y}-\frac{4 \pi}{\lambda} T^{k} \alpha(x, y)\right)
$$

$\gamma_{x . y}$ is used to represent an estimate of an unknown coherent value. After the elevation correction and deformation velocity increment of adjacent points are obtained, the absolute velocity and elevation difference of each permanent scatterer can be obtained by integrating them. At the same time, the phase unwrapping of each point is completed, and the real differential phase of each point is obtained.

It is assumed that when SAR image is acquired, the atmosphere is uncorrelated in time domain and correlated in space domain, so that low-pass filtering and high pass filtering in time domain can separate the atmospheric signal from other components of the residual (i.e. nonlinear deformation and random noise),

In the original permanent scatterer technology, the atmospheric delay phase and orbital residual phase are regarded as the same components, and they are considered to be smooth in a certain range of space, showing a linear trend in the range and azimuth $\mathrm{d}_{\mathrm{irection}}$. The equations related to the range and azimuth position are used to fit.

Then, the high pass filter in time domain is used to remove the time-dependent deformation component from the residual phase. Finally, the low pass filter in space domain is used to remove the random noise in the residual phase. The formula is as follows:

$$
\phi_{x, \text { atmo }}^{k}=\left[\left[e_{x}^{k^{\prime}}\right]_{H P_{-} \text {time }}\right]_{L P_{-} \text {space }}+\left[\overline{e_{x}}\right]_{L P_{-} \text {space }}
$$

Where, $\phi_{x, \text { atmo }}^{k}$ is the estimated atmospheric phase of the first permanent scattering point in the interferogram. According to experience, the filtering window in time domain is 300 days, and the mean filtering window in space domain is 300 days.

\section{RESEARCH AREA OVERVIEW}

In recent years, the areas with severe urban land subsidence are mainly distributed in Tianjin, Beijing, Hebei, and Shanghai. Beijing stretches across $115^{\circ} 25^{\prime} \sim 117^{\circ} 30^{\prime}$ E and $39^{\circ} 26^{\prime}$ $\sim 41^{\circ} 30^{\prime} \mathrm{N}$, and serves as the political, economic and cultural center of China. The land subsidence distribution and trend in Beijing will have an effect on urban spatial development and major engineering constructions.

For purpose of this study, the urban area of Beijing is selected as research area, covering Dongcheng District, Xicheng District, southern part of Changping District, eastern part of Haidian District, midwest part of Chaoyang District, eastern part of Fengtai District, northeast part of Daxing District, western part of Tongzhou, and a fraction of Shunyi District. These districts constitute the major urban area of Beijing, where the population is concentrated, it is economically developed, and land subsidence is relatively severe.

\section{TERRASAR IMAGE DATA PROCESSING}

\subsection{Data Processing Method}

Sequential processing and analysis were conducted on TerraSAR-X image data of 40 scenes gathered from Nov. 22, 2010 until Nov. 22, 2015 with Gamma software IPTA module (processing parameters: spatial resolution $3 \mathrm{~m}$, polarization mode $\mathrm{HH}$ and processing level L1).

\subsection{Image Space-time Baseline Analysis}

Based on analysis of time baseline, space baseline and offset of the time series InSAR data, the 20th scene (20130531) was elected as the optimal master image. Figure 1 shows the obtained space-time baseline. 


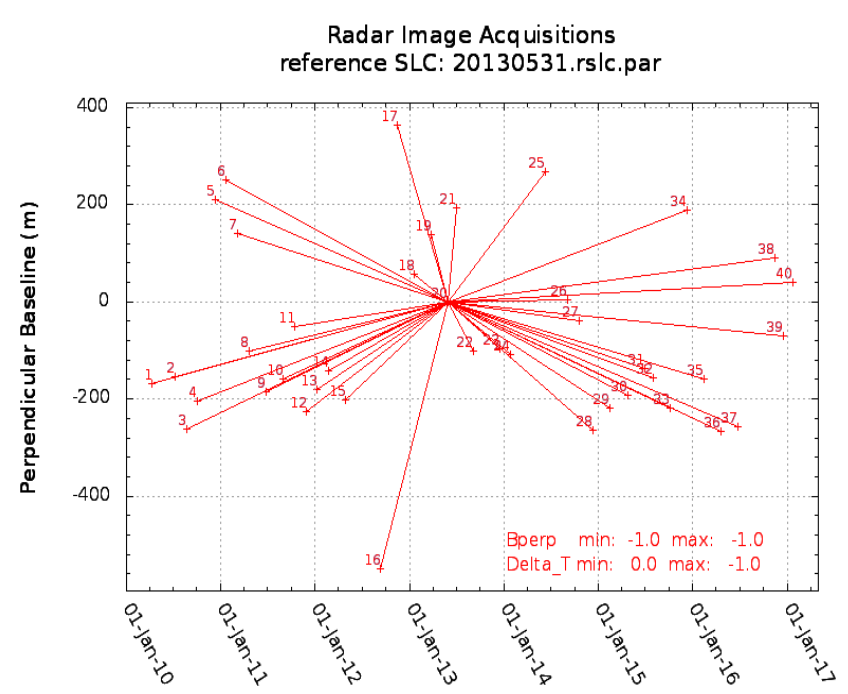

Figure 1. Image Space-time Baseline

Multi-view SAR images of a same area were studied to analyze phase and amplitude information to elect stable point targets, selection of coherence points was performed by means of coherence coefficient and amplitude deviation respectively, the candidates of selected with the two algorithms were further fused, and the duplicate points were eliminated. Time series InSAR data and 90m SRTM DEM data were georeferenced, and initial terrain phase was acquired. The selected coherence points were listed for iterative analysis, and the terrain level, mean rate of deformation, deformation history, and relative atmospheric relay of the permanent scattering points were acquired by calculating images after removing atmospheric relay phase, orbit error, terrain phase, and system noise.

\subsection{Selection of PS Point and Reference Point}

Coherence coefficient and amplitude deviation were adopted for this study. The results acquired with the two algorithms were integrated for processing to improve coherence target identification accuracy and increase coherence target quantity. Figure 2 shows the result of point selection.

871,166 candidates were selected by setting correlation threshold of spectral properties as 0.5 . High-coherence points were selected by means of amplitude deviation, and 807,413 candidates were obtained by setting amplitude deviation as 2.5 and eliminating points less than this deviation in the images. The candidates selected with the two algorithms were fused to eliminate the duplicate high-coherence points, and 1,678,579 candidates were obtained at last.
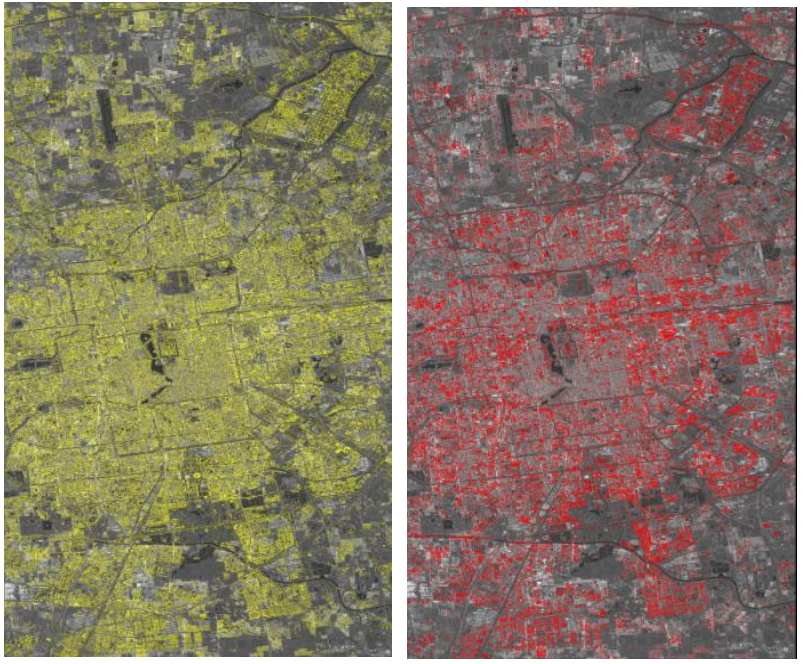

(1)Points based on spectral values

(2) Points based on amplitude deviation
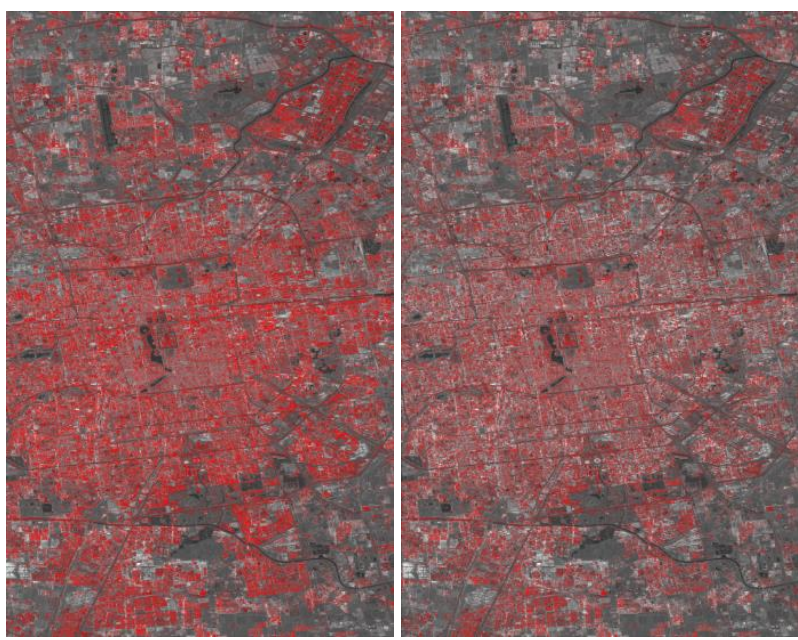

(3) Points after fusing

(4) Points after screening

Figure 2. Result of Reference Point Selection

627,937 candidates were left after eliminating the ones with sigmal value larger than 1.05. Based on relative stability analysis of various parts of the research area by sigmal value, coherence point numbered 269713 was selected as reference point. This reference point is located in a relatively stable part of the research area.

\subsection{Acquisition of Subsidence Rate}

Figure 3 shows subsidence result of urban area of Beijing by processing the screened point set with IPTA method.

The research area consists of Xicheng District, Dongcheng District, Haidian District, Changping District, Chaoyang District and portion of Daxing District.

As shown in Figure 3, relatively severe subsidence has occurred in the eastern part of Chaoyang District and southern part of Changping District, where the regional subsidence reaches up to $50 \mathrm{~mm} / \mathrm{a} \sim 80 \mathrm{~mm} / \mathrm{a}$, and Xicheng District and Dongcheng District are relatively stable. 


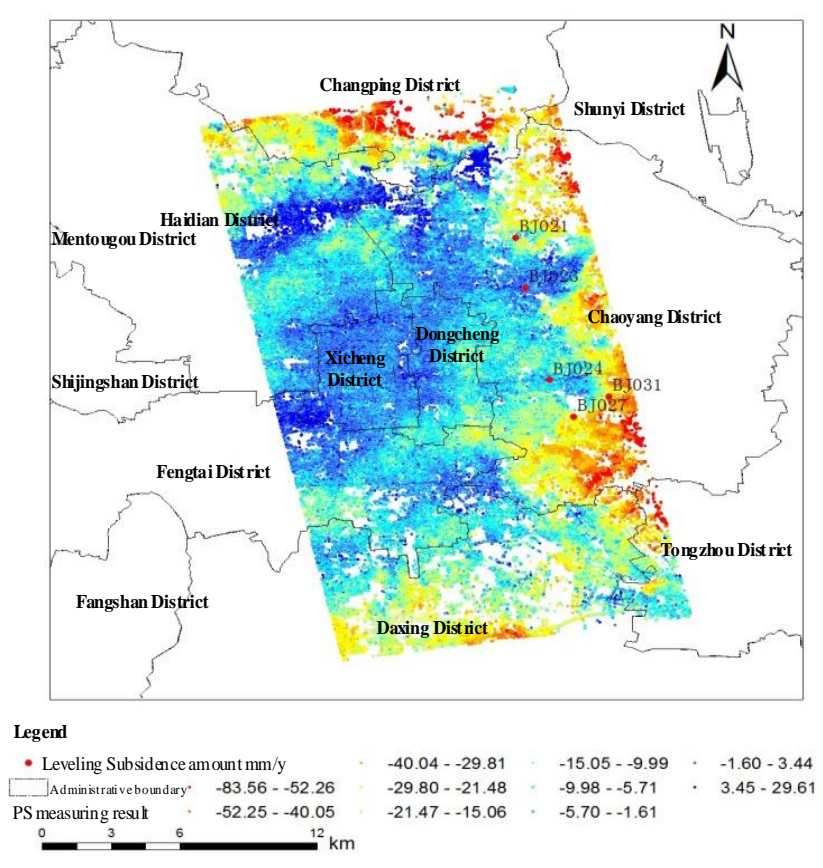

Figure 3. Subsidence Rate of Different Parts

\section{SUBSIDENCE RESULT ACCURACY EVALUATION}

To verify the reliability, comparison was made between the second-class leveling result in 2011 with PS-InSAR measuring result.

Five leveling points (BI021, BJ023, BJ024, BJ027, BJ031) in the research area were taken as basis. A circular buffer zone with a radius of $100 \mathrm{~m}$ and with the aforementioned leveling point as center was built for each leveling point, the mean value of subsidence values of all PS points in the buffer zone was set as InSAR measuring result, and leveling result and InSAR measuring result of the zone were acquired, as shown in Table 2; on this basis, calculations show the maximum absolute error between InSAR measuring result and leveling result is $8.87 \mathrm{~mm}$, the minimum absolute error is $0.21 \mathrm{~mm}$, and the standard error of the five leveling points is $3.22 \mathrm{~mm}$.

The required accuracy of absolute deviation below $10 \mathrm{~mm} / \mathrm{year}$ and standard deviation below $6 \mathrm{~mm} /$ year is satisfied. Therefore, a high accuracy can be achieved by acquiring deformation result by means of coherence point time series analysis.

Table 1. InSAR Result Accuracy Verification

\begin{tabular}{cccc}
\hline NO & $\begin{array}{c}\text { level survey } \\
(\mathrm{mm} / \mathrm{a})\end{array}$ & $\begin{array}{c}\text { InSAR } \\
(\mathrm{mm} / \mathrm{a})\end{array}$ & $\begin{array}{c}\text { absolute error } \\
(\mathrm{mm} / \mathrm{a})\end{array}$ \\
\hline BJ021 & -9.3 & -9.09 & 0.21 \\
BJ023 & -5.7 & -0.55 & 5.15 \\
BJ024 & -12.7 & -4.21 & 8.49 \\
BJ027 & -34.8 & -26.99 & 7.81 \\
BJ031 & -44.4 & -35.53 & 8.87 \\
\hline
\end{tabular}

\section{ANALYSIS OF RELATIONSHIP BETWEEN URBAN DEFORMATION AND FAULT ZONE}

\subsection{Regional Deformation Analysis of Beijing}

Four PS points were selected from Xicheng District, Shunyi District, Daxing District and Tongzhou District respectively, and the mean values of all PS points within the area 50 meters around the selected PS points in the four districts were taken for time series analysis.
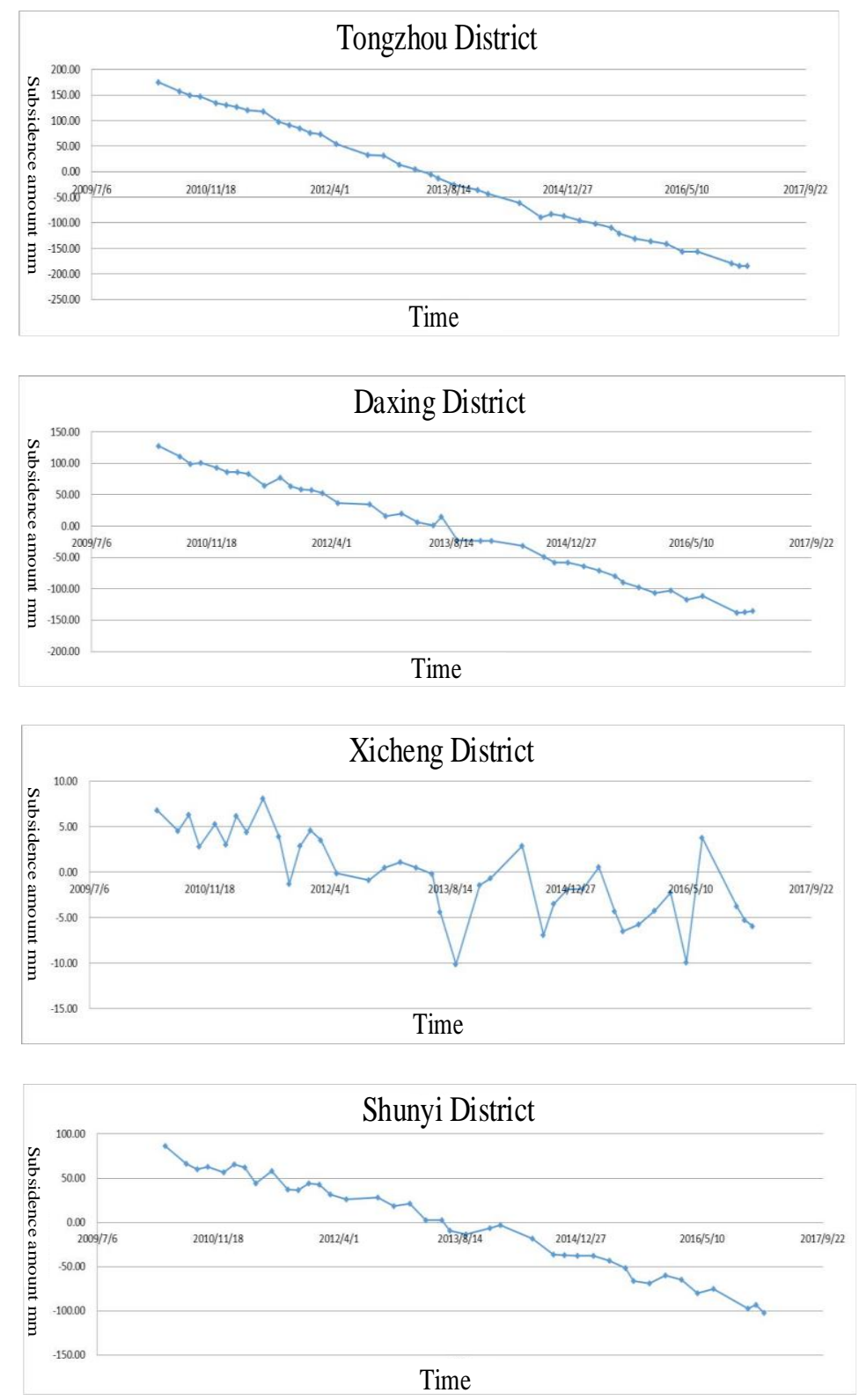

Figure 4. Time-dependent Variation Trend of Subsidence Value of PS Points in the Four Districts

Within the coverage area of image data, severe subsidence has occurred in both Tongzhou District and Daxing District. With passage of time, the subsidence value of the PS points keeps on increasing. The subsidence variations from April 2010 until Jan. 2017 are $160 \mathrm{~mm} \sim-186 \mathrm{~mm}$ (Tongzhou) and $127 \mathrm{~mm} \sim-181 \mathrm{~mm}$ (Daxing) respectively. 
The subsidence in Xicheng District takes on a relatively stable volatility with passage of time in a range of $-10.15 \sim 8.09 \mathrm{~mm}$. The subsidence in Shunyi District keeps on declining at a rate of $26.8 \mathrm{~mm} / \mathrm{a}$ by $86 \mathrm{~mm} \sim-102 \mathrm{~mm}$ in total.

As shown in Figure 4, the subsidence variation in the southern part of Changping District is $-22 \mathrm{~mm} / \mathrm{a} \sim-67 \mathrm{~mm} / \mathrm{a}$, the research area is on the edge of the subsidence funnel and takes on a trend of expanding to the north region; the subsidence variation in Haidian District is $-1 \mathrm{~mm} / \mathrm{a} \sim-22 \mathrm{~mm} / \mathrm{a}$, and the subsidence trend in the image area is gradually progressive from south to north; the subsidence variation in Dongcheng District, Xicheng District and mid-eastern Fengtai District is $-1.02 \mathrm{~mm} / \mathrm{a} \sim-6 \mathrm{~mm} / \mathrm{a}$, and shows no obvious trend, and the earth's surface is relatively stable; the subsidence variation in the northern part of Daxing District is $-3 \mathrm{~mm} / \mathrm{a} \sim-29 \mathrm{~mm} / \mathrm{a}$, a small part in the southeast of the research area has suffered from severe subsidence at a rate up to $-37 \mathrm{~mm} / \mathrm{a}$, and it might be on the edge of the subsidence funnel and shows signs of expanding to the south; the subsidence variation in Chaoyang District is $-3 \mathrm{~mm} / \mathrm{a} \sim-67 \mathrm{~mm} / \mathrm{a}$, and it is obviously shown in the figure that the middle part of Chaoyang District is on the edge of the subsidence funnel and this district has a large area suffering from subsidence, which is relatively serious; the subsidence variations in Tongzhou District and Shunyi District are $-6 \mathrm{~mm} / \mathrm{a} \sim-67 \mathrm{~mm} / \mathrm{a}$ and $-11 \mathrm{~mm} / \mathrm{a} \sim-22 \mathrm{~mm} / \mathrm{a}$ respectively, and the subsidence in Tongzhou District is relatively severe.

\subsection{Analysis of Relationship between Land Subsidence and Fault Zone}

As shown in Figure 5, there is no obvious spatial relationship between most faulted structures and land subsidence. NankouSunhe fault zone intersects with Babaoshan fault zone and Huangzhuang-Gaoliying fault zone respectively in the southern part of Changping District. This area has suffered from severe subsidence, and is considerably developed, which affects the distribution of land subsidence to a certain extent.

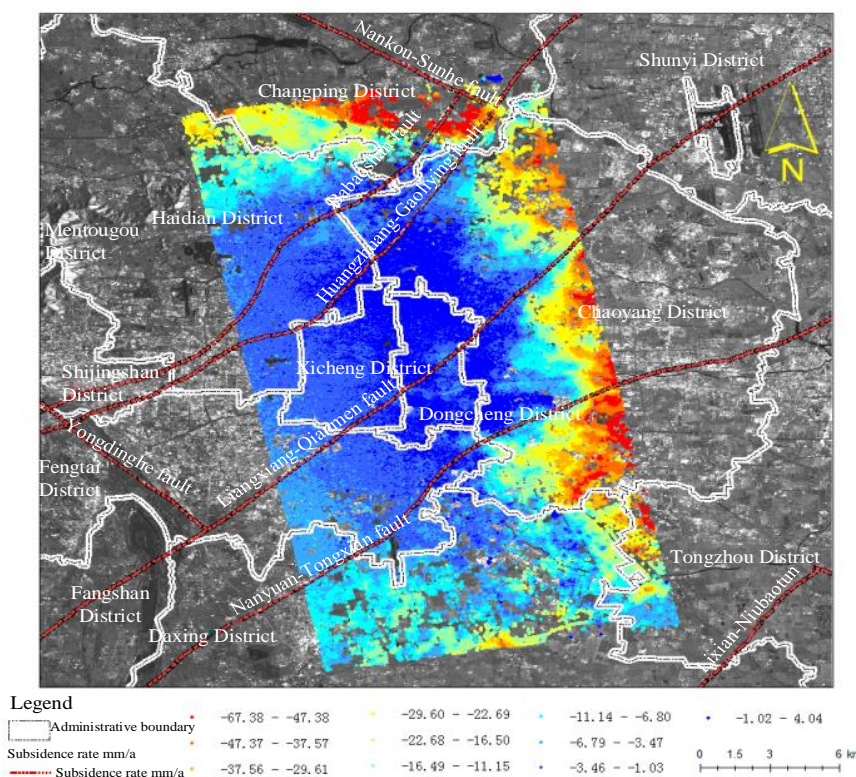

Figure 5. Overlay Chart of PS Results and Fault Zones

At the quaternary depression in Shahe Town of Changping District, the spatial distribution pattern of subsidence funnel depends on Nankou-Sunhe fault zone. The main cause of land subsidence in this area is over exploration of quaternary underground water.

Under the action of overlying load, effective stress increases to compress the aquitard pores, to lead to land subsidence. The difference in compressible stratum thickness on the two sides of the fault in this area is the main cause of large deformation gradient. Meanwhile, the regional rise is partially caused by slide of upper and lower walls of fault. Cross-section (AA' ${ }^{\prime}$ ) analysis of Nankou-Sunhe fault shows that the deformation gradient of or nearby the fault is as large as $12 \mathrm{~mm} / 1.5 \mathrm{~km}$, and the difference in annual subsidence rate on the two sides of the fault is about $5.3 \mathrm{~mm} / \mathrm{yr}$ (Figure 6).

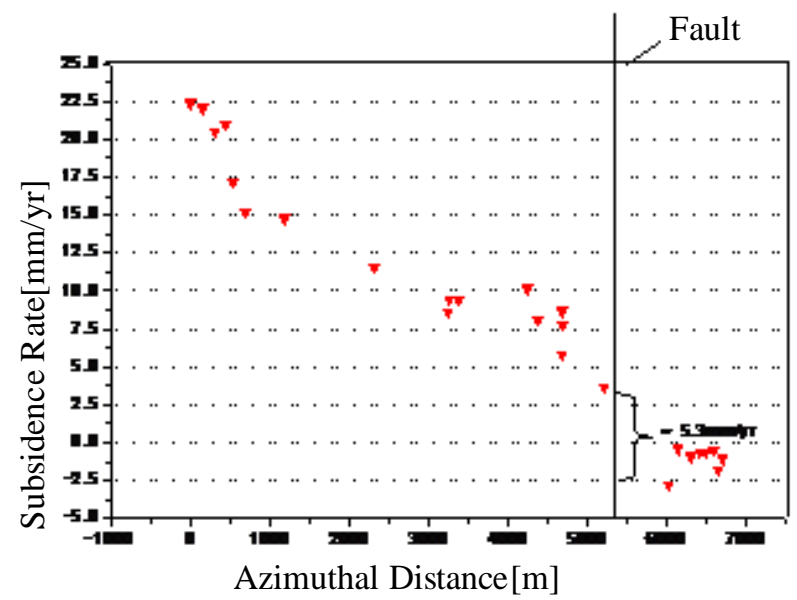

Figure 6: Annual Subsidence Rate on the Two Sides of the Fault

\section{CONCLUSION AND OUTLOOK}

The PS point subsidence data of the urban area of Beijing from Nov. 2010 until Nov. 2015 is acquired with PS-InSAR technology, accuracy evaluation is performed on the leveling results and InSAR subsidence data of the area, analysis is made on the characteristics and variation trend of land subsidence, and the spatial relationship between fault zone and land subsidence is investigated. Research shows that 1) the selection of PS points is completed by fusing the two algorithms of coherence coefficient and amplitude deviation; 2) InSAR subsidence data can clearly reveal the temporal subsidence situation nearby the points and is consistent with the leveling results. 3) Changping District, Haidian District, Daxing District and Chaoyang District all have suffered from severe subsidence; Dongcheng District, Xicheng District, and mid-eastern Fengtai District show no obvious subsidence trend and the earth's surface in these districts are relatively stable; the variations in Tongzhou District and Shunyi District are $-6 \mathrm{~mm} / \mathrm{a} \sim-67 \mathrm{~mm} / \mathrm{a}$ and $-11 \mathrm{~mm} / \mathrm{a} \sim-22 \mathrm{~mm} / \mathrm{a}$ respectively, indicating that the subsidence in Tongzhou District is relatively severe. 4) Except for the southern part of Changping District, there exists no obvious spatial relationship between fault zone and land subsidence.

In absence of leveling data of sufficient time and space resolution of the research area and ground angle reflector monitoring device, there still exist defects in deep fusion analysis of wide-range InSAR and ground monitoring data. If elevation change time series of ground GNSS CORS or geoidal surface in the same time and space of InSAR can be acquired, it will further promote the 
deep fusion of ground-to-air elevation change data, to provide technical solutions and basic methods for large-scale application in future.

\section{ACKNOWLEDGEMENTS}

This work was supported by the National Key Research and Development Program of China (No. 2016YFB0501405) \& National Natural Science Foundation of China (No. 41804038) $\&$ National Natural Science Foundation of China (No. 41504020).

\section{REFERENCE}

XIONG Peng , ZUO Xiao-qin , LI Yong-fa , et al. Application of dual-polarized Sentinel-1 data to subsidence monitoring in Kunming. Progress inGeophysics ( in Chinese), 2020 , 35(4) : 1317-1322, doi: 10. 6038 / pg2020EE0066.

XIONG Jiacheng, NIE Yunju, LUO Yue, LI Yongfei. Monitoring urban land subsidence by dual-polarization Sentinel1 data: a case study of Shanghai[J]. Bulletin of Surveying and Mapping, 2019, 0(11): 98-102,129.

XIAO Weifeng, YANG Wentao, LI Chaokui, et al. Detection of Influence Factors and Their Interaction of Land Subsidence inBeijing Based on PS-InSAR, Geomatics World,2020,27(06):713.

Galloway D L,Jones D R,Ingebritsen S E.Land Subsidence in the United States.United States Geological Survey Circular 1182[R].Reston, Virginia,1999.

Jia Sanman, Tian Fang, Qi Qian. Urban "chronic diseases"-the cause of land subsidence and comprehensive prevention and control measures.City and Disaster Reduction,2019(3):22-27.

GAO Ertao,FANDonglin,FU Bolin et al. Land Subsidence Monitoring of Nanjing Area Based on PS-InSAR and SBAS Technology[J]. jgg, 2019, 39(2): 158-163.

Wang Yimei, Luo Xiaojun, Yu Bing, et al. Monitoring ground subsidence in Zhengzhou with InSAR[J].Science of Surveying and Mapping,2019,44(09):100-106.

Wang, Xiaoqing, Zhang Peng, et al. Mitigation Atmospheric Effects in Interferogram With Using Integrated MERIS/MODIS Date and A Case Study Over Southern CaliforniaL: TheInternationalArchivesofthe Photogrammetry, RemoteSensing andSpatialInformationSciences, Volume XLII3, 2018

Yang Q, Ke Y, Zhang D, et al. Multi-scale analysis of the relationship between land subsidence and buildings: A case study in an eastern Beijing Urban Area using the PS-InSAR technique[J]. Remote Sensing, 2018, 10(7): 1006.

Solari L, Ciampalini A, Raspini F, et al. PSInSAR analysis in the Pisa urban area (Italy): A case study of subsidence related to stratigraphical factors and urbanization[J]. Remote Sensing, 2016, 8(2): 120.

Zhu L, Gong H, Li X, et al. Land subsidence due to groundwater withdrawal in the northern Beijing plain, China[J]. Engineering Geology, 2015, 193: 243-255.
WANG Xiao-qing, DANG Ya-min, ZHANG Peng, et a1 . Application of MERIS atmospheric water vapor to ASARinterferogram correction. Progress in Geophysics . (in Chinese), 2012, 27(6) : 2335 2341, doi : 10.6038/ j. issn-1004-2903. 2012. 06. 008. 